\title{
A prospective investigation into the nutritional status of 517 Irish oncology outpatients: prevalence of malnutrition, cachexia, sarcopenia and impact on quality of life
}

\author{
S.J. Cushen ${ }^{1}$, D.G. Power ${ }^{2}$, P. McEneaney ${ }^{3}$, L. Daly ${ }^{1}$ and A.M. Ryan ${ }^{1}$ \\ ${ }^{1}$ School of Food and Nutritional Sciences, University College Cork, Republic of Ireland, ${ }^{2}$ Dept Medical Oncology, \\ the Mercy University Hospital, Republic of Ireland and ${ }^{3}$ Dept of Radiology, the Mercy University Hospital, Cork, \\ Republic of Ireland
}

This abstract was awarded the prize for best Nutrition Society Irish Section oral original communication.

Malnutrition is a significant factor in predicting cancer patients' quality of life (QoL), tolerance to treatment, and overall survival ${ }^{(1)}$. Our study describes prevalence of malnutrition, cachexia and sarcopenia and their impact on QoL for the first time in Irish cancer patients undergoing chemotherapy in a regional cancer centre.

A prospective cross-sectional study of adult cancer patients undergoing chemotherapy was conducted. Malnutrition Universal Screening Tool scores (MUST) and QoL (EORTC QLQ-C30) were measured. Cancer cachexia was defined as weight loss (WL) $>5 \%$ over the past 6 months or $\mathrm{WL}>2 \%$ in combination with a body mass index $(\mathrm{BMI})<20 \mathrm{~kg} / \mathrm{m}^{2}$ or sarcopenia $^{(2)}$. Skeletal muscle was measured by computed tomography $(\mathrm{CT})$ scan in all patients. Sarcopenia was defined using published cut offs ${ }^{(3)}$.

517 patients receiving chemotherapy $(58.4 \%$ male), with a mean age of 61.8 years $( \pm 10.5 \mathrm{SD})$ were included. The most common sites of primary tumour were; colorectal (30\%), upper gastrointesntinal (GI) (23\%), and lung (12\%). 42.1\% were treated with curative intent. $53 \%$ were overweight or obese (BMI $\left.\geqslant 25 \mathrm{~kg} / \mathrm{m}^{2}\right)$. Unintentional weight loss (WL) was reported by 321 patients $(62 \%)$, with 19 $\%$ reporting WL of $>10 \%$ in the previous 6 months. Patients with tumours of the upper gut, lung and gynecological organs had the highest rates of WL. Based on CT assessment of body composition, sarcopenia was present in $47 \cdot 3 \%$ and cancer cachexia (CC) was present in $46 \%$. Sarcopenia and CC were present across all BMI categories. In the obese category $32.6 \%$ met the criteria for CC and $22.8 \%$ were sarcopenic. When nutritional risk was assessed using the MUST, $45.1 \%$ of patients were classified as 'medium' to 'high' nutritional risk, and the remainder, (284 patients, 54.9\%) were classified as 'low' nutritional risk. However, $12 \%$ of these 'low risk' patients had CC and $48.4 \%$ were sarcopenic. High C-reactive protein (CRP $\geqslant 10 \mathrm{mg} / \mathrm{L})$ was present in $47.5 \%$ of all patients. Quality of life scores differed according to tumour location, with breast cancer patients having the lowest global QoL score at $60 \pm 23 \cdot 3$ and colorectal cancer patients having the highest score a 69.2 $\pm 19 \cdot 3$. Gynecological cancers scored the lowest across all tumour types for physical, role, social and cognitive functioning scores. Cancer Cachexia, MUST and sarcopenia all demonstrated direct and significant correlations with poorer global QoL.

A significant proportion of Irish cancer patients undergoing chemotherapy experience sarcopenia and CC, however identification of malnourished patients presents challenges in the era of obesity. Site of primary tumour appears to be associated with significant weight loss, nutritional risk and QoL. Common screening tools, like the MUST, failed to identify any nutritional risk in nearly half of patients with sarcopenia. Early screening by CT assessment of body composition would allow for prompt nutrition intervention in these patients.

1. Jacquelin-Ravel N \& Pichard C (2012) Crit Rev Oncol Hematol 84, 37-46.

2. Fearon K, Strasser F, Anker SD et al. (2011) Lancet Oncol 12, 489-495.

3. Mourtzakis M, Prado CM, Lieffers JR et al. (2008) Appl Physiol Nutr Metab 33, 997-1006. 